\title{
Article
}

\section{Banking the unbanked: the Mzansi intervention in South Africa:}

Kostov, Phillip, Arun, Thankom Gopinath and Annim, Samuel Kobina Available at http://clok.uclan.ac.uk/11127/

Kostov, Phillip ORCID: 0000-0002-4899-3908, Arun, Thankom Gopinath and Annim, Samuel Kobina (2014) Banking the unbanked: the Mzansi intervention in South Africa:. Indian Growth and Development Review, 7 (2). ISSN 17538254

It is advisable to refer to the publisher's version if you intend to cite from the work. http://dx.doi.org/10.1108/IGDR-11-2012-0046

For more information about UCLan's research in this area go to http://www.uclan.ac.uk/researchgroups/ and search for < name of research Group>.

For information about Research generally at UCLan please go to http://www.uclan.ac.uk/research/

All outputs in CLoK are protected by Intellectual Property Rights law, including Copyright law. Copyright, IPR and Moral Rights for the works on this site are retained by the individual authors and/or other copyright owners. Terms and conditions for use of this material are defined in the policies page.

\section{CLoK}

Central Lancashire online Knowledge www.clok.uclan.ac.uk

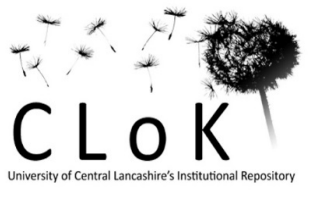


This is a preprint to paper in Indian Growth and Development Review, 7(2), http://dx.doi.org/10.1108/IGDR-11-2012-0046

\title{
Banking the unbanked: the Mzansi intervention in South Africa
}

\begin{abstract}
This paper aims to understand household's latent behaviour decision making in accessing financial services. In this analysis we look at the determinants of the choice of the pre-entry Mzansi account by consumers in South Africa.

We use 102 variables, grouped in the following categories: basic literacy, understanding financial terms, targets for financial advice, desired financial education and financial perception. Employing a computationally efficient variable selection algorithm we study which variables can satisfactorily explain the choice of a Mzansi account.

The Mzansi intervention is appealing to individuals with basic but insufficient financial education. Aspirations seem to be very influential in revealing the choice of financial services and to this end Mzansi is perceived as a pre-entry account not meeting the aspirations of individuals aiming to climb up the financial services ladder. We find that Mzansi holders view the account mainly as a vehicle for receiving payments, but on the other hand are debt-averse and inclined to save. Hence although there is at present no concrete evidence that the Mzansi intervention increases access to finance via diversification (i.e. by recruiting customers into higher level accounts and services) our analysis shows that this is very likely
\end{abstract}

\section{Introduction}

There is a large body of substantial theoretical and empirical literature discussing and measuring the importance of efficient financial systems for long-term economic development (see Levine 2005 for a survey). Furthermore financial development is generally accepted to exert beneficial effects on both growth and poverty alleviation (see e.g. Beck et al., 2007). While traditionally empirical studies (generally in cross-country setting) have focused on financial depth, recently analytical attention have turned towards the issues of financial outreach and inclusion, something often referred to as access to 
This is a preprint to paper in Indian Growth and Development Review, 7(2), http://dx.doi.org/10.1108/IGDR-11-2012-0046

finance. Recently The World Bank Economic Review published a special issue dedicated to this topic (WBER, 2008, vol. 22, no. 3). The issue of barriers to financial services has become an emerging topic in the development literature, both from a macro-economic perspective and from the general micro-finance literature. The implicit assumption of this argument is that the supply side constraint is the interesting subject of analytical attention. This is either because one may not be able to directly observe the latent demand for financial products whenever the supply constraints are in place or because "those who have access but choose not to use services pose less of a problem for policymakers” (Beck and Demirguc-Kunt, 2008). Relaxing the supply-side barriers would however bring the potential lack of demand for financial services to the forefront with important policy implications. After all coordinated policy intervention designed to expand access to finance is costly and such costs will need to be compared to the potential benefits and voluntary nonparticipation, when the barriers to access are removed, reduces these benefits. Hence studying the determinants of demand for financial services becomes an important research question. In this type of analysis the important question becomes whether exclusion from financial services is voluntary or involuntary. It is clearly infeasible to try to carry out reliable distinction between these two forms of exclusion in the presence of binding barriers established at the supply side. There are however two cases where such investigations could be conducted. One is when individuals are transferred into a different environment where such barriers are not implemented. For example Osili and Paulson (2008) examined the determinants of financial market participation among immigrants in the USA and found that institutional factors have profound effects. They hypothesised that the main channel through which these institutional factors affect market participation are beliefs. Beliefs however are difficult to measure and in a more general framework can be represented by alternative indicators such as values, attitudes, perceptions and levels of financial literacy. We argue that these have to be considered when evaluating the potential effect of expanding access to finance. The uptake of new financial services bridging over existing barriers will define who will benefit the expanded access to finance.

Investigations such as this of Osili and Paulson (2008) are important in that they open a totally new research agenda and allow characterisation of voluntary participation in accessing financial services. Note however that although such studies are highly informative 
This is a preprint to paper in Indian Growth and Development Review, 7(2), http://dx.doi.org/10.1108/IGDR-11-2012-0046

in guiding possible policy interventions and outlining the boundaries of future research into these issues, they nevertheless suffer from an important drawback. Transplanting individuals from one environment to a totally different one may tell us a lot about attitudes and culture, but it does not sufficiently well capture the institutional framework established in their home country. It would be much more useful if the decision to avail to different forms of financial services could be investigated in a more natural environment, i.e. in a developing country context. This is where the second case in which such investigation could be conducted arises. It is when barriers to financial access are lowered or removed in a concerted policy intervention in a developing country. Studying such financial choices in the latter case could be very useful in better characterising what defines financial behaviour and due to the natural setting of such a study it can provide even more useful guides for future policy interventions.

This paper takes a preliminary step in this research quest considering the case of the Mzansi intervention in South Africa. The Mzansi intervention represents a coordinated roll-out of financial services in targeting the poor and vulnerable. Classical cases of such increased access to finance are interventions from microfinance institutions and private commercial banks on one hand or through legislation and state owned banks pioneering expansion in access to financial services. Rarely, is the experience of an intervention that draws on a linkage between private commercial banks and either state-owned banks or an act of legislation to expand financial access. Furthermore the co-ordinated and wide-ranging scope of the Mzansi intervention provides an opportunity to study the individual financial choice in a developing context without the presence of binding supply side restrictions.

\section{Background}

Until the end of the apartheid era in 1994, financial services in South Africa were predominantly accessible to the non-black community and therefore by default excluded the economically weaker households (Meagher and Wilkinson 2006). In South Africa, the 'Mzansi' account intervention offers a peculiar case in which commercial banks and a state owned financial institution takes a lead role to expand access to financial services. Finmark Trust (2009) cautions that although significant successful indicators are identifiable with the 
This is a preprint to paper in Indian Growth and Development Review, 7(2), http://dx.doi.org/10.1108/IGDR-11-2012-0046

'Mzansi' account intervention replicating this strategy require similar country level socioeconomic and financial landscape characteristics. Also the evidence of 1.6 million accounts classified as dormant within the first four years of the Mzansi account operation underscores the importance of circumspection and identification of the drivers and perceptions of household's decision to open and maintain an accounts. This paper aims to understand household's latent behaviour and decision making in accessing financial services.

Following the Financial Service Charter (FSC) policy recommendations the 'Mzansi' account which was initiated in 2004 offered entry-level accounts. The FSC was a consensus reached by the four main private banks in South Africa (Absa, Nedbank, First National and Standard) and a Government-owned financial institution (Postbank). Although it was open (voluntary) other private financial institutions did not join possibly due to different market niches other than targeting the unserved. The intervention was situated in the broader context of financial services outreach that is scale ${ }^{1}$, depth ${ }^{2}$, scope ${ }^{3}$ and breadth ${ }^{4}$. Operating under the broader government policy framework of economically empowering the black community in South Africa, the FSC aimed at providing effective access to transaction and savings financial services. While the initiative was not legally backing, consenting financial institutions, through some form of social contract were committed to expanding access and providing affordable financial services to the wider population. Beyond national goals, participating institutions, four main private banks in South Africa (mentioned above) and a state-owned financial institution (Postbank) were motivated based on a consensus of shared reputational and financial risk.

In terms of depth of outreach, the specific target of the issuing institutions was that $80 \%$ of the fifth segment of the South African living standard population ${ }^{5}$ should have effective access to transaction and savings products and services. From a supply perspective effective access was defined in the context of proximity, non-discriminatory, affordability, product diversity and understandability. Based on bank performance in the South African context

\footnotetext{
${ }^{1}$ Scale means reaching more clients in terms of numbers.

${ }^{2}$ Depth refers to Targeting poorer clients.

${ }^{3}$ Scope is defined as offering a wide range of product/services and diversified strategies including physical and electronic services.

${ }^{4}$ Breadth means reaching clients with different socio-demographic characteristics especially vulnerable groups.

${ }^{5}$ The fifth segment is the bulk of poor households at the bottom of the poverty pyramid.
} 
This is a preprint to paper in Indian Growth and Development Review, 7(2), http://dx.doi.org/10.1108/IGDR-11-2012-0046

and comparable ratios in other developing countries, breadth of outreach for the Mzansi account was targeted at 2,173,930 ${ }^{6}$ by 31st December 2008.

Products offered by each of the issuing banks were standardized and dispensed at a relatively lower cost. The common minimum products offered included; issuance of debit card; absence of monthly administration fee; ceilings on balances; KYC-driven (Know Your Customer) ceilings on transaction value; restrictions on certain electronic payments services; and no difference between withdrawals on a bank's own ATM ('on us') and withdrawals using another bank’s ATM ('not-on-us’) (Finmark Trust, 2009).

Monitoring the experience of Mzansi account initiative after the fourth year of its inception, Finmark Trust 2009 shows that the number of accounts opened between October 2004 and December 2008 was 6.0 million. In the context of the South African adult population, a tenth of the population has an Mzansi account. While this figure appear convincing about two-fifth of the accounts were inactive. Outcomes of the Mzansi intervention in terms of depth of outreach showed that $74 \%$ of Mzansi active account holders had income levels below USD200 compared to $41 \%$ of the currently banked population excluding Mzansi. Also from the perspective breadth of outreach, for instance rural-urban location, more than half of the Mzansi active account holders were located in rural areas. Beyond the outreach dimensions of access to financial services, the Mzansi account initiative has received popular reception among all stakeholders especially the political ones.

In spite of the achievements, threats by some of the issuing institutions to drop out of the collaborative initiative (supply) and the significant number (42 per cent) of inactive accounts (demand) require attention. In this paper, we concentrate on the demand side issues. We investigate household inherent motivation in choosing a financial service. In particular, their financial education background (how financially literate they are), opinions on their financial needs, aspirations and attitudes. Our central research question is - in what way do these hidden factors (education, perception and attitudes) affect the uptake of Mzansi account?

\footnotetext{
${ }^{6}$ This target was applicable to only the private banks since the government entity was not a signatory to the Charter.
} 
This is a preprint to paper in Indian Growth and Development Review, 7(2), http://dx.doi.org/10.1108/IGDR-11-2012-0046

\section{Conceptual Framework}

While a plethora of evidence exists on the supply-side conditions (product design, market conditions and regulation) required for expanding access to financial services (Claessens 2006 and Beck et al 2009), actual demand has lately been questioned (Anand and Rosenberg 2008). Contextualizing access to finance individuals can be classified into four broad categories. The first group consists of those who need financial services and are able to access them. Secondly, we identify those who need financial access but are not able to obtain it due to supply-side constrains such as rigid conditions and distance. In contrast to the second group are those who are unable to access financial services due to demand-side constrains such as financial illiteracy, poor attitude and stereotypes on financial services and credit in particular. The final group is made of individuals who simply do not need financial services irrespective of either the supply-side or the demand-side constrains. This does not imply that those in the last group cannot access any financial services, but rather that they do not want to use the formal sector providing them and could instead rely on informal arrangements.

Discourse on access to finance has mainly thrived on the assumption that all individuals fall into either the first or the second category. Empirical studies that limit the scope of questions to say 'did households receive credit in the past year or not' and without probes on willingness to access financial services, unconsciously force clients into either of the two categories. Also, the emerging advocacy for financial literacy and consumer education, attest the case of presumption that individuals need access to finance but are not equipped with its prudential usage. The forgoing suggests a potential distinction between poor household's interest in engaging in an economic activity and willingness to access a financial service. That is, the decision to engage in an economic activity does not necessarily translate into need for financial services. As Osili and Paulson (2008) discuss institutional factors are important in defining whether such access is actively sought. Since institutions are essentially behavioural rules and routines, then behavioural determinants will have profound effect on the decision whether to access or not available financial services.

The attitude and perception of individuals on financial services places them in either the third or fourth category. Analysing the attitude and perception of individual access to 
This is a preprint to paper in Indian Growth and Development Review, 7(2), http://dx.doi.org/10.1108/IGDR-11-2012-0046

financial services is often blurred with the issue of attributing constraints to either supplyside or demand-side. The problem of identification worsens in the case where constraints are emerging from both the supply and demand sides of access to financial services. From pragmatic perspective, this can be addressed through a sequential approach. South Africa's approach of extensively addressing supply-side constraints from all three dimensions of product design, market conditions and regulation offers a suitable platform for demand-side investigation. Understanding the drivers of individuals' willingness and ability to take-up a financial service will help redirect policy that will aid prudent decisions on access to financial services and demystify unfounded stereotypes about financial service providers' rigidities, exclusivity, bureaucracies, high cost of service and barriers of entry.

\section{Mzansi's Role in Access to Finance}

Classens (2006) identifies four pre-requisites for improved access to financial services, namely; availability, reliability, flexibility and continuity of access to financial services. Two of the four requirements that are availability and reliability can be identified with the 'Mzansi' account initiative. Firstly, the worth and reputation of the four commercial banks coupled with the credibility of the state bank naturally invokes confidence of sustainability and non-exploitation in potential clients. Secondly the widespread coverage of all the issuing institutions especially Postbank, presents potential clients with proximity of a financial service point of transaction either physically or electronically.

Broadly speaking the overall aims of the Mzansi intervention seem to have been reached, although the financial sustainability of the scheme and therefore its attractiveness to banks could be questioned (Finmark Trust, 2009). In particular the depth and breadth of the programme have exceeded expectations. The behavioural and attitudinal constraints however have not been explored in detail. Initial evaluations of the Mzansi intervention have focused on directly inquiring clients about their opinion and attitudes. Thus we know what socio-demographic characteristics the Mzansi account holders possess and what their declared intensions are ((Finmark Trust, 2009). The mode of elicitation of their motivation however could condition their stated opinion. To better understand what truly makes a customer go for a Mzansi account, one needs to dig deeper. Hence instead of simply 
This is a preprint to paper in Indian Growth and Development Review, 7(2), http://dx.doi.org/10.1108/IGDR-11-2012-0046

analysing the differences between Mzansi holders and non-holders, it is preferable to investigate the choice of Mzansi conditional on their background. Conventional financial wisdom would dictate that the relevant background variables are those measuring who they are. When access to financial services is concerned, these include their financial literacy, i.e. the elements of their financial 'education' and understanding of financial concepts. These will differentiate the actual from the stated scope of the Mzansi intervention. Note however that simple comparison of the distribution of these characteristics between holders and nonholders can to a large extent capture this. The internal motivation of clients could however be measured by their general attitude towards financial literacy, namely what they think is missing from their financial understanding and what they want to learn. Providing characterisation of the Mzansi choice based on these internal motivation would not simply complement the 'who they are' characterisation, but would also describe in some more detail the realised scope of the Mzansi intervention. Therefore such an analysis would contribute towards a better fine tuning of the actual scope of Mzansi, so that it better reflects the needs of its customers. This could have twofold benefits. First it could go some way in addressing the issue of high share of inactive Mzansi accounts. Second it would undoubtedly help banks better target their clients by better differentiating Mzansi from their entry level products and hence contributing to achieving a longer term sustainability of the scheme.

In this paper we conceptualise the choice of financial services in terms of perceptions, attitudes and beliefs. Note that all the above are essentially behavioural determinants. Therefore we take a broader institutional view of the analytical issue. Since institutions are basically behavioural routines, institutional factors can also be subsumed in this analytical framework. Note however that we explicitly exclude from consideration, such 'natural' potential determinants of the access to finance such as e.g. age, income, work status, location etc. Such an omission could look like a clear misspecification to an average applied economist. Note however that in our broad conceptual framework, such characteristics will define the perceptions, attitudes and beliefs. An individual acts to access a certain financial product, not because he/she is of a certain age, but because this individual holds some values (beliefs, attitudes, perceptions) that lead him/her to chose or reject this particular product. It could be expected that because of the relationship between such background variable and the behavioural determinants used in this study, the former will impact on the 
This is a preprint to paper in Indian Growth and Development Review, 7(2), http://dx.doi.org/10.1108/IGDR-11-2012-0046

latter and hence would be correlated with the choice. Such a relationship is however indirect and therefore could lead to misleading conclusions. If we were using a wish-list approach, then we could have included such variables in a structured hierarchical type of model where both direct and indirect impacts would be specified. Note however that this would greatly increase the complexity of our task. When there is no compelling theoretical background or readily available empirical results of how the choice of financial services is carried out, we need a preliminary analysis. In undertaking such a preliminary study under conditions of large uncertainty about the possible determinants, it is sufficient to map only the relevant behavioural determinants. When this is done, one may easily complement the model by adding the discussed background variable explaining the selected reduced set of behavioural variables, if one wishes to do so.

\section{Data}

The empirical section of the paper relies on the 2007 FinScope $^{7}$ South Africa dataset. The sample frame of the FinScope data consists of all South Africans aged 16 years and above. The nationally representative data is based on enumeration areas and stratified and multistage sampling technique. The 2007 FinScope dataset was collected from 3900 households using face to face interviews from April to May 2007. The scope of the data was categorized under two broad headings namely, living standard and financial services. The former captures issues such as income, quality of life, and household demographics, while the latter tracks national financial access patterns and pathways in terms of products and service providers and household financial decision trees and perceptions.

In this analysis we use 102 variables, grouped in the following categories: basic literacy, understanding financial terms, targets for financial advice, financial education desired and financial perception. Table 1 provides an overview of these categories, while appendix one includes a full listing of the variables within each category.

\section{Insert Table 1}

\footnotetext{
${ }^{7}$ FinMark Trust (www.finmark.org.za) in 2003 launched FinScope (www.finscopeafrica.com) in an attempt to establish credible benchmarks for the use of, and access to, financial services in South Africa. The aim was to achieve a measure and understanding of consumer demand across transactions, savings, credit and insurance categories.
} 
This is a preprint to paper in Indian Growth and Development Review, 7(2), http://dx.doi.org/10.1108/IGDR-11-2012-0046

It is a common practice in empirical studies to consider choices conditional on different background characteristics. Such conventional empirical approach employs variables that measure who the individuals (households) are. Given the overwhelming prevalence of such practices in empirical analysis, it might look surprising that in this paper we ignore this type of variables. Here we advocate that such background variables should measure motivations and attitudes, rather than be individual descriptors. To better explain this motivation, consider the following example: should a variable like e.g. age be expected to have impact on the choice of financial services? Empirical researchers unquestionably include such variables, mainly because previous studies used them. This practice is however deeply flawed. One does not access certain financial service because of his/hers age per se, but because of perceptions and beliefs about his/hers own personal needs.

Such perceptions may be (and could be expected to be) socially conditioned. Hence it is logical that via processes of dynamic learning, peer pressure etc age can be correlated with such core beliefs and values. Then because of the confounding variable effect the empirical regression is likely to find correlation between age and financial choices. Yet such finding is simply a correlation artefact due to the confounding effect of the missing variables (measuring such beliefs and values). It would however be naive to hypothesise direct causation, which the practice of including such a variable into a regression type of model implicitly assumes. Furthermore explaining choices in terms of attitudes and perception provides a more interpretable and logically structured explanation of financial choices. Such an approach is actually better linked to economic theory that the background variables inclusion practice. Economic theory postulated that individual choices a based on subjective (expected) utility and since belief and values are direct measures of this subjectivity, they are clearly relevant to such choices. Most 'background' variables clearly are not.

The internal motivation of clients could be measured by their general attitude towards financial literacy, namely what they think is missing from their financial understanding and what they want to learn. Providing characterisation of the Mzansi choice based on these internal motivations would not simply complement the 'who they are' characterisation, but would also describe in some more detail the realised scope of the Mzansi intervention. 
This is a preprint to paper in Indian Growth and Development Review, 7(2), http://dx.doi.org/10.1108/IGDR-11-2012-0046

One van nevertheless notice that we omit a background variable that is related to demand for financial services, namely income. Income measures the transactional demand for financial access and hence is directly contributing to it. As explained above, the main focus of this investigation are the demand side constraints to access to finance, i.e. the beliefs and attitudes that create voluntary exclusion form financial services. For this reason it is preferable to concentrate on potential determinates of financial exclusion. Furthermore there are some purely technical considerations. The effect of income is expected to be highly nonlinear and would be difficult to deal with is a generalised linear model, as the one used here.

Here we will only briefly describe these categories. The understanding of financial terms category includes 17 variables that quantify whether the respondents comprehend the actual meaning of a number of financial terms, ranging from simple ones such as bad debt and loan to increasingly more complex ones such as pieces of financial legislation. In principle these variables can be considered to have a natural ordering. One could hypothesis that clients would need some basic financial understanding in order to select themselves into the Mzansi intervention, but having better understanding would mean that they would not be satisfied with the basic features offered by Mzansi and would require more sophisticated products.

The target for financial advice category includes 15 variables asking whether the respondents would use certain sources in order to obtain financial advice. The sources range from informal ones (such as family and friends) to formal professional type of advice providers (such as financial institutions and independent brokers). This category complements the financial education variables in that it measures certain attitudes towards finance. It demonstrates the confidence respondents have in their own understanding of financial matters, but also measures their attitude and trust in different sources of financial advice. It is difficult to elaborate on what one should expect with respect to this category, since it does not seem to be a clear hierarchy in the ordering of the financial advice sources. Furthermore, some of the sources could be formulated in such a way that respondents may not properly understand them and/or different types of advice sources could be bundled into the same category (e.g. "A financial advisor other than an independent broker (e.g. tax consultant, auditor)”).

Further 16 variables describe what type of financial education respondents desire. These variables imply the respondents do not possess this form of financial education. Most 
This is a preprint to paper in Indian Growth and Development Review, 7(2), http://dx.doi.org/10.1108/IGDR-11-2012-0046

importantly however they measure their intrinsic aspirations to further themselves. In this way this category complements the financial education category in a very important and significant way. People aspiring to obtain further financial education could aspire to move up the financial access ladder. Therefore even if their background (financial education) places them on a particular step of this ladder, their aspirations motivate them and hence they have incentive to move up. In this way they may end up at a higher step, simply because this is where they (aspire to) belong. Hence this category measures and quantifies the inherent motivations of banking clients. With respect to Mzansi, which is essentially a pre-entry level account, one could hypothesise that most types of expressed desire to obtain further financial education would move clients up towards the banks' entry level accounts and hence have negative impact on Mzansi uptake.

Finally we have two categories of financial perceptions variables. These ask people whether they agree with some statements. In this way the internal motivations and perceptions towards financial behaviour are evaluated. The financial perceptions grid 1 variables ask factual questions about actual behaviour, while grid 2 variables refer mostly to attitudes (opinion, trust etc.). Thus the two categories of financial perception complement each other by measuring two facets of the same phenomenon.

\section{Methodology}

In the particular problem we are facing here, our dataset consist entirely of factor variables. Therefore a binary dependent variable type of model is a natural choice for the modelling framework. In particular the logistic regression is a common choice for modelling in such situations. Here we will implement a logistic regression explaining the choices (from an individual point of view) of the Mzansi intervention. Logistic regression belongs to the class of generalised linear models (McCullagh \& Nelder, 1989). In this particular instance however there is high degree of uncertainty about which variables belong to the model. Our general conceptual framework provides us with a large number of potential explanatory variables (102 in this case). It would however be impractical to run a regression of the whole set of potential explanatory variables. 
This is a preprint to paper in Indian Growth and Development Review, 7(2), http://dx.doi.org/10.1108/IGDR-11-2012-0046

It is not uncommon in applied economics one to either run multiple regressions and discuss which of these is more appropriate or to present a single regression model with large number of variables, and discuss which are statistically significant, and which are not. This practice has two major drawbacks. Everyone who has run regressions with large number of variables knows well that dropping insignificant variables could change coefficient estimates, significance or even signs for the remaining variables. Hence when variables that do not belong to the model are included one is estimating the wrong model and therefore the subsequent inference would be biased and unreliable. Therefore it is important that the proper statistical model is used for inference. There are cases when underlying theoretical models can provide guidance on which variables need to be included in such models. The problem with empirical work is that even when an underlying model is available, the datasets may exclude some of the required variable or alternatively provide different proxies for unobservable or difficult to measure variables. Consequently often a researcher has to face the issue of having a potentially large number of explanatory variables to be included in a model. Then the questions of primary interests are which variables are to be included in the model (variable selection, model selection) and what are the parameters pertaining to the variables that belong to the model (model estimation). As discussed above these two questions are interrelated.

The issue we are facing here requires determination of the relevant variables and estimation of their effects. Tibshirani (1996) introduced the Least Absolute Shrinkage and Selection Operator (LASSO) designed for simultaneous shrinkage and variable selection in linear models. In simple terms LASSO uses the $L 1$ (sum of absolute deviations) constraint in the regularization step. That is, the estimator is calculated by minimizing the relevant empirical risk function subject to the constraint that the $L 1$ norm of the regression coefficients is bounded (by a given positive number). An equivalent and much more popular way to define the LASSO estimator is as penalised estimator, in which the relevant empirical risk function is augmented by a penalty term proportional to the L1 norm of the regression coefficients. Formally the LASSO can be expressed as:

$$
\hat{\beta}(\lambda)=\arg \min _{\beta}\left\{\ell(\beta)+\lambda \sum_{j=1}^{p}\left|\beta_{j}\right|\right\} \text {, }
$$


This is a preprint to paper in Indian Growth and Development Review, 7(2), http://dx.doi.org/10.1108/IGDR-11-2012-0046

where $\ell(\beta)$ is the corresponding loss function (in this case the negative log-likelihood for the logistic regression model), $\mathrm{p}$ is the number of parameters (to be estimated), and $\lambda$ is the penalty applied to the L1 norm of the coefficients. The penalty term shrinks the coefficients towards zero. Larger penalty terms achieve greater shrinkage, while smaller penalties induce less shrinkage. Unlike the ridge estimator (which uses L2 norm penalty), the LASSO achieves sparse solutions, (i.e. some coefficients become exactly zero) which is a useful property when variable selection is desired. The presence of the penalty term in the optimisation problem above introduces some bias, but reduces the variance of the estoiimator, hence triggering the variance-bias trade-off, which effectively depends on the choice of the penalty term (i.e. $\lambda$ ). An appropriate choice of $\lambda$ can achieve optimality in terms of the variance-bias trade-off. Different methods for such optimal choice have been suggested in the literature. Here we will sue cross-validation.

The latter LASSO approach have been subsequently extended to a wide range of models such as e.g. wavelets (Chen et al., 1999), kernel machines (Gunn and Kandola, 2002; Roth, 2004), smoothing splines (Zhang et al., 2003), multiclass logistic regressions (Krishnapuram et al., 2004) etc. These models extend the standard LASSO in two important directions namely by introducing new loss functions (other than the squared loss) and increasing the number of the $L 1$ constraints applied in the regularisation step. Hereafter we will refer to all these models simply as LASSO models. Although being consistent in terms of variable selection in that it retains the important variables, the original LASSO estimator applies fixed amount of shrinkages to all coefficients, which can be a problem when the so called oracle property is desired. In particular the LASSO estimator can be an oracle one only under some special circumstances subject to non-trivial conditions (see Zou, 2006 for details). In simple terms an estimator possessing the oracle property will have the same asymptotic distribution for the coefficient estimates as the 'oracle' estimator, or in other words the estimator implemented knowing which coefficients are zero. This allows an oracle estimator to be used not only for variable selection, but also for inference. Nevertheless when an adaptive amount of shrinkage for each regression coefficient is 
This is a preprint to paper in Indian Growth and Development Review, 7(2), http://dx.doi.org/10.1108/IGDR-11-2012-0046

implemented this leads to estimators which possess the oracle property (Zou, 2006; Wang et al., 2007). Fortunately the application of the adaptive LASSO can be implemented using the same algorithms as the standard LASSO. To explain this consider the LASSO estimator:

$\widehat{\beta}(\lambda)=\arg \min _{\beta}\left\{\ell(\beta)+\lambda \sum_{j=1}^{p}\left|\beta_{j}\right|\right\}$

where $\ell(\beta)$ is the corresponding loss function (in this case the negative log-likelihood for the logistic regression model), $p$ is the number of parameters (to be estimated), while $\lambda$ is a (fixed) non-negative penalty parameter describing the amount of shrinkage applied to the coefficients. The adaptive LASSO can be defined as:

$$
\hat{\beta}_{\text {adapt }}(\lambda)=\arg \min _{\beta}\left\{\ell(\beta)+\lambda_{\text {init }} \lambda_{\text {adapt }} \sum_{j=1}^{p} \frac{\left|\beta_{j}\right|}{\left|\tilde{\beta}_{j, \text { init }}\right|}\right\}
$$

where $\tilde{\beta}_{j, \text { init }}$ is an initial estimate and $\lambda_{\text {init }}$ is the penalty applied in obtaining this initial estimate. If the initial estimate is unpenalised, we set $\lambda_{\text {init }}=1$. In essence the adaptive LASSO is a two-stage estimator, the second stage of which has the standard LASSO form. This could be more easily seen if we rewrite the adaptive LASSO estimator as:

$$
\hat{\beta}_{\text {adapt }}(\lambda)=\arg \min _{\beta}\left\{\ell(\beta)+\lambda_{\text {adapt }} \sum_{j=1}^{p}\left(\frac{\lambda_{\text {init }}}{\left|\tilde{\beta}_{j, \text { init }}\right|}\left|\beta_{j}\right|\right)\right\}
$$

which is clearly a LASSO problem with adaptive amount of shrinkage applied to the coefficients, but it is also a standard LASSO estimator that can be calculated using the standard LASSO algorithms.

The first stage could be a LASSO estimate, but could also be an unpenalised logistic regression (in which case $\lambda_{\text {init }}=1$ ). In this paper for simplicity we will follow the latter option for obtaining initial values, mainly to reduce the computational costs.

The implementation of the LASSO in efficient algorithms is of particular interest when larger high dimensional datasets are involved. Tibshirani (1996) used the quadratic programming method for least squares regressions and a combination of iteratively reweighted least squares with quadratic programming for logistic regressions. Osborne et al. 
This is a preprint to paper in Indian Growth and Development Review, 7(2), http://dx.doi.org/10.1108/IGDR-11-2012-0046

(2000) proposed a faster quadratic programming algorithm for linear regressions, which was extended for logistic regressions by Lokhorst et al. (1999) and Roth (2004). Efron et al. (2004) developed a computationally more efficient algorithm called LARS to calculate the whole regularisation path for linear regressions. Wang and Leng (2007) proposed a method of least squares approximation to translate many different types of LASSO objective functions into their asymptotically equivalent least-squares problems. This allows application of the LARS algorithm to the transformed problem and therefore allows estimation of non-linear models, such as e.g. logistic regression. The above algorithms, however, are not easily applicable to large dimensional data sets. The reason for this is that they apply repeated matrix inversions the computing costs for which increase rapidly with the dimension of the dataset. Additionally when there are singularities in the design matrix, something that is quite likely in highly correlated real datasets, the matrices constructed inside the algorithms could be singular, leading to general convergence failure. Since the theoretical properties for regularisation estimators are established with regard to the optimal amount of shrinkage, the quality of the estimated regularisation path estimates are crucial is selecting the right amount of shrinkage. Perkins et al. (2003) proposed a stagewise gradient descent algorithm which they called grafting. The grafting algorithm avoids the matrix inversion, but requires high dimensional nonlinear constraint optimisation inside the iterations, which would be computationally demanding. Grandvalet and Canu (1999) implemented a fixed point algorithm based on the equivalence between the adaptive ridge regression and LASSO. The problem with latter is that it is not guaranteed to achieve global convergence.

Here we instead focus on the coordinate descent methods. Different versions of these methods have been proposed for the LASSO a number of times, but only recently were their power and computational capabilities fully appreciated. Early references include Fu (1998), Shevade and Keerthi (2003) and Daubechies et al. (2004) and more recently Friedman et al. (2007) and $\mathrm{Wu}$ and Lange (2008). The theoretical properties of coordinate descent algorithms are presented in Tseng (2001). Here we will employ the gradient algorithm of Kim et al. (2008). It combines a coordinate-wise descent algorithm with a deletion step to solve the LASSO problem. This gradient LASSO approach uses coordinate-wise descent to add variables to the model (thus avoiding matrix inversion). Its novelty compared to the conventional coordinate descent methods is the addition of a deletion step that adaptively 
This is a preprint to paper in Indian Growth and Development Review, 7(2), http://dx.doi.org/10.1108/IGDR-11-2012-0046

removes variables in order to improve convergence. The deletion step avoids the slow convergence properties of the conventional coordinate descent algorithms near the optimum and by reducing the number of steps helps to increase the overall speed.

The results, reported in this paper were obtained using the free statistical language $R$ ( $R$ Core Team, 2013). For computational efficiency reasons the gradient adaptive LASSO algorithm used C code due to Kim et al. (2008), interfaced to and callable from within R. The source C code for the gradient LASSO (and the R driver for it) have been downloaded from the JCGS webpage. The adaptive LASSO is technically a weighted version of the LASSO estimator and as such can be implemented by supplying appropriate weights. These have been derived by an initial run of a standard logistic regression. The penalty was chosen by 5 -fold cross-validation. The stability selection uses author written $\mathrm{R}$ code, while the probability value calculations rely on the R package hdlm (Arnold, 2012) with plugged in adaptive gradient LASSO estimation.

\section{Results}

We apply the gradient LASSO algorithm to compute the whole regularisation part for the logistic regression model of the choice of Mzansi account on the set of 102 variables, described in the data section.

The stability selection uses author written $\mathrm{R}$ code, while the probability value calculations rely on the $\mathrm{R}$ package hdlm (Arnold, 2012) with plugged in adaptive gradient LASSO estimation.

The algorithms for regularised estimation (such as the adaptive LASSO used here) do not directly provide an easy way to derive standard errors. Tibshirani (1996) provided an asymptotic standard error formula for the LASSO. Its asymptotic nature can however introduce uncertainty bias. Although not applicable to our case, other approaches have also been suggested. E.g. Fan and $\mathrm{Li}$ (2001) demonstrated that the local quadratic approximation (LQA) can provide a sandwich formula for computing the covariance of the penalized estimates of the nonzero components which is consistent (Fan and Peng 2004). 
This is a preprint to paper in Indian Growth and Development Review, 7(2), http://dx.doi.org/10.1108/IGDR-11-2012-0046

By far the simplest way to obtain standard errors is to simply run a standard (logistic) regression with only the selected variables. Such an approach relies upon the oracle property of the estimators (which meant that its asymptotic distribution is the same as that of an oracle estimator. This is what is known as the post-LASSO estimator (see Belloni and Chernozhukov, 2013 for the liner models case) and has been applied in high dimensional model selection (see e.g. Kostov, 2010 for a non-regularisation implementation). Such an approach is feasible and easy to implement. However any inference based on it would be conditional of the result of the variable selection method. The oracle property is however an asymptotic property. In other words if one implements the estimator (adaptive LASSO in this case) many times, on average it will select the right variables and their distribution would (asymptotically) converge to that of the oracle. In a single run however any oracle estimator can omit important variables or include unimportant ones.

It is therefore useful if the model uncertainty due to that fact that the estimator is only applied once is also accounted for. While this is rather straightforward for Bayesian approaches, it is somewhat more complicated here. Here we report two different measures of model uncertainty.

First we report uncertainty adjusted probability values. In essence we use the data splitting approach of Wasserman and Roeder (2009), but instead of the single split, apply the multiple split proposal of Meinshausen et al. (2009) with 10 splits. This allows us to produce a standard regression table. For reporting purposes we include the probability value, (instead of the standard errors or the t-statistics) since it is easier to interpret in terms of variables significance. Furthermore we applied the subsampling based stability selection approach of Meinshausen and Buehlmann (2010) to evaluate the model inclusion probability for each variable, as a complementary uncertainty measure.

The results, reported in this paper were obtained using the free statistical language $R$ ( $R$ Core Team, 2013). For computational efficiency reasons the gradient adaptive LASSO algorithm used C code due to Kim et al. (2008), interfaced to and callable from within R. The source C code for the gradient LASSO (and the R driver for it) have been downloaded from the Journal of Computational and Graphical Statistics webpage. The adaptive LASSO is technically a weighted version of the LASSO estimator and as such can be implemented by supplying appropriate weights. These have been derived by an initial run of a standard 
This is a preprint to paper in Indian Growth and Development Review, 7(2), http://dx.doi.org/10.1108/IGDR-11-2012-0046

logistic regression. The penalty was chosen by 5-fold cross-validation, with logistic loss estimated alongside the path to choose the optimal amount of shrinkage for the adaptive LASSO estimator.

Table 2 presents the selected independent variables (i.e. the variables with non-zero coefficients) and their estimated coefficients, together with their p-values and inclusion probabilities.

\section{Insert Table 2}

Out of the 102 candidate variable, only 13 are retained in the estimated model. Below we discuss the obtained results.

First, the basic literacy variable does not affect the choice of Mzansi. Since basic literacy can be expected to be a pre-requisite to any form of access to finance, it could be expected to be able to discriminate between individuals with and without such access. Note however that since the individuals without Mzansi comprise of both people lacking access and people who use better than the basic Mzansi access, such a variable cannot differentiate Mzansi holders from the rest.

Next, four variables from the "understanding of financial terms" category are retained. These are Bad debt, Ombudsman, Counselling about debt and Garnishee order or emolument order. After taking into account the model uncertainty, these are all highly significant and characterised by high inclusion probability. Bearing in mind that this category has an almost natural hierarchy from simpler to more complex terms, the selected terms lie at the lower end of this hierarchy. Therefore the individuals selecting themselves into the Mzansi system only possess some understanding of basic financial terms, i.e. have a minimal financial education. They need such basic understanding in order to be motivated to seek access to finance. Had however they possessed better financial knowledge, they would have opted for more advanced forms of access, from the basic entry accounts upwards. The above explanation is further confirmed by the estimated coefficients. Note that since our variables are indicators (and therefore are expressed in the same 'units') we can directly compare their coefficients in terms of magnitude because the size of the coefficients in this case is directly proportional to the relative effect of the corresponding variable. To this end the understanding of the term Ombudsman, which indicates a higher level of financial literacy that the other three selected variables shows considerably smaller impact (because 
This is a preprint to paper in Indian Growth and Development Review, 7(2), http://dx.doi.org/10.1108/IGDR-11-2012-0046

its coefficient is much smaller). The two variables: Bad debt and Counselling about the debt, which are clearly related since they both are related to debt, capture most of the magnitude of the effect in this category. This shows that Mzansi account holders are aware about debt and this is a defining characteristic of people opting into the Mzansi intervention. This demonstrates a desire to undertake some kind of financial planning about the future. Note however that the coefficients estimated for the variables in this category are rather small, both in principle and relative to the other categories in table 2. This shows that such effects are relatively small in term of relative magnitude. Since these results indicate a distinction 'from below' i.e. compared to individuals that do not possess any access to financial services (which comprises of $26 \%$ of the population in South Africa), it is nevertheless an important result, particularly when situated in the broader discourse of access to finance.

No variable from the "targets for financial advice" category is selected. This suggests that the Mzansi account holders are not that different from the rest of the sample in what refers to their preferred choice of financial advice. Alternatively, since the sample contains both individuals with no financial access and such with enhanced (compared to Mzansi) access to finance, the model finds it difficult to explicitly differentiate between differences that could be attributable to these two alternative categories. A major reason for this could be the fact that in contrast to the "understanding of financial terms" category, here it is difficult to find similar natural hierarchy, which means that the differentiation of the choice of Mzansi from below (i.e. against no access) and above (i.e. better access) are pooled together. The latter was not a problem with the previous category due to the natural hierarchy, but in terms of the sources of financial advice, the lack of such confuses matters. Furthermore it is possible that the wording of the options within this category could be confusing for respondents.

The next category is "financial education desired". Three variables from this category feature in our model. First of all seeking no further financial education makes one more likely to choose Mzansi. Mzansi holders have reached a level of financial literacy, but do not strive to move further, they are happy where they are. There are two interesting features of this result. First this is the far the largest, in terms of magnitude, coefficient in the model. However, after accounting for the uncertainty, this coefficient is actually insignificant, although marginally so. The inclusion probability is however (slightly) over $50 \%$. The 
This is a preprint to paper in Indian Growth and Development Review, 7(2), http://dx.doi.org/10.1108/IGDR-11-2012-0046

above suggests that the effect of this variable is potentially very strong, but only supported on a compact (small) subset of the sample. In other words, here we apply a variable selection approach which aggregates the effects for each variable and as a result suggests that this effect is not very significant. If instead one has opted for a model averaging approach, where the inclusion and exclusion densities for the relevant coefficients are threated separately, the conclusion would have been rather different. One possible explanation for the above results is that not seeking further financial education can also mean that such individuals already possess it, in which case they would not require Mzansi. Therefore since this statement may have two different interpretations with expected opposing effects on the choice of Mzansi, variable selection approach would not be able to properly distinguish between them.

The other two selected variables in this category, namely effective usage of technology and working out how much credit can be afforded or paid back, make decision to choose Mzansi less likely. Both these variables are highly significant and have inclusion probabilities of $100 \%$. The effects are to be expected, because looking for further financial education expresses desire and aspirations to move further up the financial access ladder. This is confirmed by the type of these two variables. The first one relates to the effective use of technology (hence moving into other financial access channels such as e.g. mobile banking). The other variable relates to working out credit repayments. This could show a desire to overcome debt, but also maybe planning for minor investments. This is consistent with the results from the "understanding of financial terms" category, where debt issues and a revealed attitude towards some sort of financial planning are evident. What is also highly informative is which variables (from this category) are not selected. In particular most of these refer to basic financial literacy such as e.g. understanding interest rates. As discussed above such variables cannot effectively discriminate between Mzansi holders and individuals with no financial access. The financial aspirations can distinguish Mzansi from above, but not from below, and most of these omitted variables are essentially measuring such low level aspirations. The finding that it is not simply characteristics, but also aspirations that define the choice of channels for access to finance is however highly significant. 
This is a preprint to paper in Indian Growth and Development Review, 7(2), http://dx.doi.org/10.1108/IGDR-11-2012-0046

The next two categories are the financial perceptions. These are grouped in two grids. Grid one measures what we tentatively call revealed perceptions. These show an interesting picture. The first selected variable states that Mzansi holders generally agree with the statement (i.e. individuals agreeing with this statement are more likely to choose Mzansi) that money is to be withdrawn as soon as possible. This is consistent with the known fact that many Mzansi accounts are used mainly for receiving payments. Furthermore this variable has the second largest coefficient amongst all selected variables. This is not only consistent with the fact that large number of Mzansi accounts are used simply as device for receiving money for immediate withdrawal, but also quantifies that attitudes that lead to this type of behaviour are dominant amongst the Mzansi account holders. The important implication of the latter is that it questions the sustainability of the Mzansi intervention, as applied at the present. The other two variables in this category ("You go without basic things so that you can save" and "You have a will or last testament") however tell a rather interesting story. The first one reveals a preference towards saving and debt aversion amongst Mzansi holders. In a country where $72 \%$ of the adult population do not save, this is a very important finding. As such it suggests that not everything is doom and gloom with Mzansi. It provides a hint that it could, in principle become sustainable for the banks from financial point of view. Note however, that this would only be possible if these preferences become realised, i.e. if a sufficient share of the Mzansi population can afford to save and reduce indebtedness, which in turn depends on a number of other factors such as e.g. economic growth etc. Taking into account the general awareness of debt issues, as demonstrated from the results for the other categories, this provides an indication that there are segments of the Mzansi intervention where sustainability (from banks' point of view) could be achieved. This however would require more targeted approach, so different from the present broad brush one, that it will unquestionably restrict access to finance. It is of course possible for banks to conduct such focused targeting within the present form of the Mzansi intervention. For the latter they will need to take into account the available information about the behaviour and the attitudes of their customers and use this to identify the potential clusters of sustainability.

The last selected variable in this category states that having a will makes the choice of Mzansi less likely. This comes as no surprise since this indicates a higher level of financial planning, consistent with level of resources that define higher level of financial access. 
This is a preprint to paper in Indian Growth and Development Review, 7(2), http://dx.doi.org/10.1108/IGDR-11-2012-0046

What could be slightly surprising is why the algorithm chooses this particular variable to distinguish the Mzansi choice 'from above'. Since the variables included so far (and discussed above) reveal a tendency toward some kind of basic financial planning, this particular variable provides an upper (as opposed to the lower in the other similar variables) bound to the financial planning type of indicators for Mzansi.

The second grid measures stated, as opposed to acted upon (i.e. elicitated from their actual behaviour), perceptions in the first grid. The nature of these questions may create an elicitation problem in the sense that they can be easily misinterpreted. Therefore the results for this category have to be treated with caution. There are three variables from this category that are retained in the model. The first fact to note is that all these variables have rather small (in terms of magnitude) coefficients, which indicates that in relative terms these variables are not as important. Looking at the variables themselves, the first one refers to the statement that young people know more about money than older ones. Agreeing with this statement increases the chances for opting into Mzansi. It is difficult to ascertain the precise meaning of this result, particularly given its small coefficient and the possibility of an elicitation problem. Taking into account the recent surge in technological innovations in banking, e.g. mobile phone banking, electronic cards etc, a type of technology which older people naturally find more difficult to cope with, this may denote a belief that you need to be able to 'understand' such technological changes to deal with money. If on the other hand one thinks older people are better this would lead to more 'traditional' attitude. 'Traditional' in South Africa where 28\% of adults receive money from friends and family as a source of income, could imply no formal access to financial services. The other variable refers to having trust in informal associations. Although significant, it only has a $61 \%$ inclusion probability. Having no trust in informal institutions reduces the probability to have a Mzansi account. Trust is an integral element of any kind of access to financial services, be they formal or informal. Therefore one can view informal associations as a type of 'introduction' into the word of financial services and its mechanisms and characteristics. When one does not have trust in their procedures, such mistrust would naturally extend to the more formal types of access to finance. Note however that the above assertion would not be universally applicable. In more traditional societies, where trust is primarily rooted in tradition, family and friends it will start with informal associations before progression into more formal channels, as we argue here. In more 'modern' societies on the other hand, trust 
This is a preprint to paper in Indian Growth and Development Review, 7(2), http://dx.doi.org/10.1108/IGDR-11-2012-0046

into the formal channels may be accompanied by mistrust into informal associations, which are not part of the established 'system'.

The last included variable in this category, namely 'allow monthly deduction from a cell phone account' reduces the probability of choosing Mzansi. It is somewhat surprising that a variable like that was retained and hence unclear what should be the precise interpretation of the above. Note however that the coefficient of this variable is in terms of magnitude by far the smallest one amongst all selected variables. Furthermore, although marginally this variable is insignificant and characterised by inclusion probability of less than $50 \%$ and hence would have to be rejected. This means that we should not attach too much significance to the above result. Bearing in mind the trust interpretation of the other variables in this category, this particular one may approximate some 'upper bound' on trust in that the algorithm may have found this particular variable as most correlated with such a hypothetical bound. Such an interpretation is however highly speculative and as outlined above should be regarded with considerable caution. The main reason we kept it in is the fact that the single run LASSO (incorrectly) selected this variable, and hence one can view this as an illustration of the uncertainly characterising such single run estimators. None of the variables that were omitted in the single run estimator obtained inclusion probability of $50 \%$ or higher and hence were excluded from the model.

\section{Conclusions}

This paper adds to the general discourse on access to finance in investigating the demand side determinants of participation in financial markets. Although the issue of barriers to access have been the dominant theme in development literature for quite a while, relaxing the supply-side barriers would bring the potential lack of demand for financial services to the forefront with important policy implications. After all coordinated policy intervention designed to expand access to finance is costly and such costs will need to be compared to the potential benefits and voluntary non-participation, when the barriers to access are removed, reduces these benefits. Hence studying the determinants of demand for financial services becomes an important research question. The uptake of new financial services bridging over existing barriers will define who will the expanded access to finance benefit. 
This is a preprint to paper in Indian Growth and Development Review, 7(2), http://dx.doi.org/10.1108/IGDR-11-2012-0046

This paper takes a preliminary step in this research quest considering the case of the Mzansi intervention in South Africa.

We use a large dataset of potential determinants of individual choice of a Mzansi account. The Mzansi intervention is found to be implicitly targeted at individuals with basic but insufficient financial education. It is a pre-entry account and as such it is generally perceived of not meeting the aspirations of individuals aiming to climb up the financial services ladder. We found that aspirations (i.e. who the individuals want to be) explain financial choices better that status (i.e. who they actually are) variables. The linkages from aspirations and attitudes to actual realised behaviour are however far from straightforward.

Furthermore we find that Mzansi holders on the one hand view the account mainly as a vehicle for receiving payments, but on the other hand are debt-averse and inclined to save. This suggests that the Mzansi population is rather heterogenous in terms of beliefs and other important from financial point of view characteristics. This heterogeneity suggests that researching and identifying different segments in the overall population could help better identification of their financial needs and such better targeting could increase both the effectiveness of such interventions and also ultimately lead to some form of financial sustainability of policies aimed at increase finance participation.

This is a preliminary exploratory study of a largely understudied issue and as such has a number of limitations. First, the choice of financial services is defined as a function of perception, beliefs and attitudes. This greatly increases the interpretability of the results within the adopted conceptual framework, but since such variables are typically unobservable, it may somewhat reduce the empirical value of the paper in that it may impede the formulation of detailed practical policy recommendation. Note however that it should be straightforward to complement the model with such variables, explaining the variables used in the study and this would not affect our results. Another limitation is that we do not account for dynamic factors. In an area of rapid change such are very important. The inferred model structure can however be used to further investigate such effects and in particular previous experiences. Similarly to the previous point, such effects are fairly easy to add to our framework as a form second stage investigation that builds upon our results. Finally a more detailed investigation of the problem in better defining the choice problem (e.g Mzansi or no access or Mzansi or alternative access) could be undertaken to obtain 
This is a preprint to paper in Indian Growth and Development Review, 7(2), http://dx.doi.org/10.1108/IGDR-11-2012-0046

better tuned answers. All these alternative avenues for research are not only compatible with the present approach, but can also be built upon its results.

\section{References}

Anand, M. and R. Rosenberg. (2008) AreWe Overestimating Demand for Microloans, Brief, Washington, D.C.: CGAP, April.

Arnold, T. B. (2012). hdlm: Fitting High Dimensional Linear Models. R package version 1.1.1, http://CRAN.R-project.org/package=hdlm.

Beck T. and A. Demirguc-Kunt (2008) Access to Finance: An Unfinished Agenda, The World Bank Economic Review, 22(3), 383-396.

Belloni, A and V. Chernozhukov (2013) Least squares after model selection in highdimensional sparse models, Bernoulli 19(2), 521-547.

Chen, S. S., D. L. Donoho, and M. A. Saunders (1999) Atomic decomposition by basis pursuit. SIAM Journal on Scientific Computing 20 (1), 33-61.

Daubechies I, Defrise M, De Mol C (2004) An Iterative Thresholding Algorithm for Linear Inverse Problems with a Sparsity Constraint. Communications on Pure and Applied Mathematics, 57, 1413-1457.

Efron, B., T. Hastie, I. Johnstone, and R. Tibshirani (2004) Least angle regression. Annals of Statistics 32, 407-499.

Fan, J. and R. Li (2001) Variable selection via noncocave penalized likelihood and its oracle properties. Journal of the American Statistical Association 96, 1348-1360.

.Fan, J., and H. Peng (2004), On Nonconcave Penalized Likelihood with Diverging Number of Parameters, The Annals of Statistics, 32, 928-961.

FinMark Trust (2009) The Mzansi Bank Account Initiative in South Africa, http://www.finmarktrust.org.za/documents/R_Mzansi_BFA.pdf, Accessed: March 2010.

Friedman J, Hastie T, Hoeing H, Tibshirani R (2007). Pathwise Coordinate Optimization. The Annals of Applied Statistics, 2(1), 302-332. 
This is a preprint to paper in Indian Growth and Development Review, 7(2), http://dx.doi.org/10.1108/IGDR-11-2012-0046

Fu W (1998) Penalized Regressions: The Bridge vs. the Lasso, Journal of Computational and Graphical Statistics, 7(3), 397-416.

Grandvalet, Y. and S. Canu (1999) Outcomes of the equivalence of adaptive ridge with least absolute shrinkage. In M. Kearns, S. Solla, and D. Cohn (Eds.), Advances in Neural Information Processing Systems, Volume 11, pp. 445-451. MIT press.

Gunn, S. R. and J. S. Kandola (2002) Structural modelling with sparse kernels. Machine Learning 48, 115-136.

Kim, J., Y. Kim, Y. Kim (2008) Gradient-Based Optimization Algorithm for LASSO, Journal of Computational and Graphical Statistics. 17(4): 994-1009.

Kostov, P. (2010) Model boosting for spatial weighting matrix selection in spatial lag models, Environment and Planning B: Planning and Design, 37(3), 533-549.

Krishnapuram B, Hartemink AJ (2005) Sparse Multinomial Logistic Regression: Fast Algorithms and Generalization Bounds." IEEE Transactions on Pattern Analysis and Machine Intelligence, 27(6), 957-968.

Krishnapuram, B., L. Carlin, M. Figueiredo, and A. Hartemink (2004) Learning sparse classifier: Multi-class formulation, fast algorithms and generalization bounds. Technical report, ISDS, Duke university.

Lokhorst, J., B. A. Turlach, and W. N. Venables (1999) Lasso2*: An S-plus library to solve regression problems while imposing an L1 constraint on the parameters.

Meinshausen N, L. Meier and P. Buehlmann (2009). P-values for high-dimensional regression, Journal of the American Statistical Association, 104(488), 1671-1681.

Meinshausen, N. and P. Buehlmann (2010) Stability Selection (with discussion), Journal of the Royal Statistical Society, Series B 72, 417-473.

Osborne, M. R., B. Presnell, and B. A. Turlach (2000) A new approach to variable selection in least squares problems. IMA Journal of Numerical Analysis 20 (3), 389-404.

Osili, U. O. and A. Paulson (2008) What Can We Learn about Financial Access from U.S. Immigrants? The Role of Country of Origin Institutions and Immigrant Beliefs, The World Bank Economic Review, 22(3), 431-455. 
This is a preprint to paper in Indian Growth and Development Review, 7(2), http://dx.doi.org/10.1108/IGDR-11-2012-0046

Perkins, S., K. Lacker, and J. Theiler (2003) Grafting: Fast, incremental feature selection by gradient descent in function space. Journal of Machine Learning Research 3, 1333-1356.

R Core Team (2013). R: A language and environment for statistical computing. R Foundation for Statistical Computing, Vienna, Austria. ISBN 3-900051-07-0, URL http://www.R-project.org/

Roth, V. (2004). The generalized lasso. IEEE Transactions on Neural Networks 15 (1), 1628.

Shevade K, Keerthi S (2003) A Simple and Efficient Algorithm for Gene Selection Using Sparse Logistic Regression. Bioinformatics, 19, 2246-2253.

Tibshirani, R. (1996) Regression shrinkage and selection via the lasso. Journal of the Royal Stratistical Society B, 58, 267-288.

Tseng P (2001) Convergence of a Block Coordinate Descent Method for Nondifferentiable Minimization. Journal of Optimization Theory and Applications, 109, 475-494.

Wang, H. ,R. Li and C.-L. Tsai (2007) Regression coefficient and autoregressive order shrinkage and selection via lasso. Journal of Royal Statistical Society Series B, 69, 63-78.

Wang, H. and Leng, C. (2007) Unified LASSO Estimation via Least Squares Approximation. Journal of the American Statistical Association, 102, 1039-1048.

Wasserman L and K. Roeder (2009) High dimensional variable selection, The Annals of Statistics, 37(5A), 2178-2201.

Wu T, Lange K (2008) Coordinate Descent Procedures for LASSO Penalized Regression. The Annals of Applied Statistics, 2(1), 224-244.

Zhang, H. H., G. Wahba, Y. Lin, M. Voelker, M. Ferris, R. Klein, and B. Klein (2003) Variable selection and model building via likelihood basis pursuit. Technical Report 1059r, Department of Statistics, University of Wisconsin, Madison, WI.

Zou, H. (2006), The adaptive LASSO and its oracle properties," Journal of the American Statistical Association, 101, 1418-1429. 
This is a preprint to paper in Indian Growth and Development Review, 7(2), http://dx.doi.org/10.1108/IGDR-11-2012-0046

Table1. Variables overview

$\overline{\text { Variable Category } \quad \text { Number of variables }}$

Basic Literacy 1

Understanding of financial terms $\quad 17$

$\begin{array}{ll}\text { Targets for financial advice } & 15\end{array}$

Financial education desired $\quad 16$

Financial perceptions grid $1 \quad 28$

Financial perceptions grid 2 
This is a preprint to paper in Indian Growth and Development Review, 7(2), http://dx.doi.org/10.1108/IGDR-11-2012-0046

Table 2. Gradient LASSO estimation results

\begin{tabular}{|c|c|c|c|}
\hline Variable & Coefficient & P-value & $\begin{array}{r}\text { Inclusion } \\
\text { Probability }\end{array}$ \\
\hline Intercept & 0.84 & 0.18 & \\
\hline Understanding of financial terms - Bad debt & 0.11 & 0.00 & 0.95 \\
\hline Understanding of financial terms - Ombudsman & 0.02 & 0.03 & 0.87 \\
\hline $\begin{array}{l}\text { Understanding of financial terms - Counselling } \\
\text { about debt }\end{array}$ & 0.07 & 0.01 & 0.87 \\
\hline $\begin{array}{l}\text { Understanding of financial terms - Garnishee } \\
\text { order or emolument order }\end{array}$ & 0.10 & 0.00 & 1.00 \\
\hline
\end{tabular}

Financial education desired - How to make

effective use of technology, such as cellphones

or ATMs, to better manage your finances

$-0.10 \quad 0.00$

1.00

Financial education desired - How to work out

how much credit you can afford/pay back on $\quad-0.03 \quad 0.00 \quad 1.00$

$\begin{array}{llll}\text { Financial education desired - None } & 0.38 & 0.11 & 0.52\end{array}$

Financial perceptions grid 1 - As soon as money

is deposited into your account you withdraw it $\quad \begin{array}{llll}0.36 & 0.00 & 1.00\end{array}$

Financial perceptions grid 1 - You go without

$\begin{array}{llll}\text { basic things so that you can save } & 0.10 & 0.01 & 0.83\end{array}$

Financial perceptions grid 1 - You have a will or

$\begin{array}{llll}\text { last testament } & -0.20 & 0.00 & 1.00\end{array}$

Financial perceptions grid 2 - When it comes to

money, young people know more than older

$\begin{array}{llll}\text { people } & 0.05 & 0.00 & 0.82\end{array}$

Financial perceptions grid 2 - You don’t trust

informal associations like stokvels or umgalelos

$\begin{array}{llll}\text { or savings clubs } & -0.06 & 0.03 & 0.61\end{array}$

Financial perceptions grid 2 - You would allow

$\begin{array}{llll}\text { monthly premiums or instalments to be deducted } & -0.02 & 0.13 & 0.39\end{array}$ 
This is a preprint to paper in Indian Growth and Development Review, 7(2), http://dx.doi.org/10.1108/IGDR-11-2012-0046

from your prepaid cellphone account

Note: Intercept is not subject to shrinkage. 